\title{
XVI. Energy production
}

Humans need energy to live. This is provided by food. The basal metabolism of a human being requires about $2.7 \mathrm{kWh} /$ day. Human beings need also energy to produce work, to manufacture objects, to move from one place to the other with vehicles, to produce light when it is dark, to cook food, etc. This energy is provided by various sources such as oil, natural gas, coal, biomass, hydroelectric power, solar power, wind power etc.

The availability of abundant and affordable energy is fundamental to the increasing quality of life enjoyed by the inhabitants of our planet. Since the industrial revolution, among the benefits of this has been a dramatic increase in the life expectancy of people. For example, in France, the life expectancy was less than 30 years in 1880 . It is more than 80 years today. Unfortunately, poor countries still have much smaller life expectancies than rich countries.

The first step in the energy chain is energy production from primary energy sources. At the global level, the primary energy supply amounted to 13.5 $\mathrm{Gtoe}^{12}$ in 2013. Most of the energy that we currently use comes from fossil fuels (oil, coal and natural gas) which are in finite quantities in the earth. Figure 126 shows the percentage of the different energy sources contributing to global primary (prior to any transformation) energy consumption in 2013. Fossil fuels support more than $80 \%$ of the global primary energy consumption.

While Humankind relies heavily on fossil fuels, they are in finite quantity in the earth and will be depleted on a timescale of the order of, at most, a few centuries. This is a serious constraint. Decreasing fossil fuel consumption and replacing it with sustainable energy sources is a high priority at the global level. The relative contribution of fossil fuels to the global primary energy supply has decreased from $86.7 \%$ in 1973 to $81.4 \%$ in 2013 . However, in absolute value the fossil fuel consumption has increased (from about 5,3 Gtoe in 1973 to 13.5 Gtoe in 2013).

A second major constraint facing the world is the emission of carbon dioxide $\left(\mathrm{CO}_{2}\right)$ and more generally of greenhouse gases such as methane, nitrogen oxides, etc. The $\mathrm{CO}_{2}$ emissions increase the greenhouse effect and can induce a climate change with a negative impact on the living conditions on the planet. We are basically emitting about twice as much $\mathrm{CO}_{2}$ as nature can absorb with natural processes.

Final energy corresponds to the energy available to the consumer after transformation of the primary energy (electricity, gasoline, diesel oil, 


\section{Total primary energy supply}

(2013)

\section{Other}

Hydro

Nuclear

\section{Biofuels and waste}

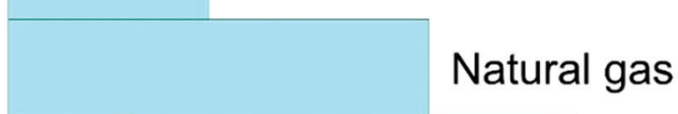

Coal/peat

Oil

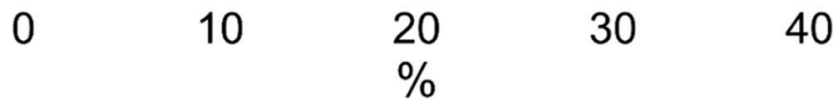

Figure 126. Sources of primary energy consumed, 2013. Other includes wind, solar, geothermal, etc. Data from www.iea.org.

purified natural gas or purified coal, etc.). The global final energy consumption in 2013 was equal to 9,3 Gtoe representing $68.7 \%$ of the total primary energy supply. This inefficiency in the energy conversion is partly due to physical laws, such as the Carnot principle, but also to yields in the different operations. The latter can be increased using innovative technology such as nanotechnology.

\section{Fossil fuels}

Crude oil has to be refined before it can be used by consumers. Gasoline, diesel oil etc. are obtained in this way. The efficiency of the refining processes and the yield are important economic issues. Nanocatalysis plays a key role in this respect since nanotechnology provides the ability to fabricate more efficient catalysts with less active material. In petroleum refining, nanocatalysis is used in the four major processes indicated in figure 127. 


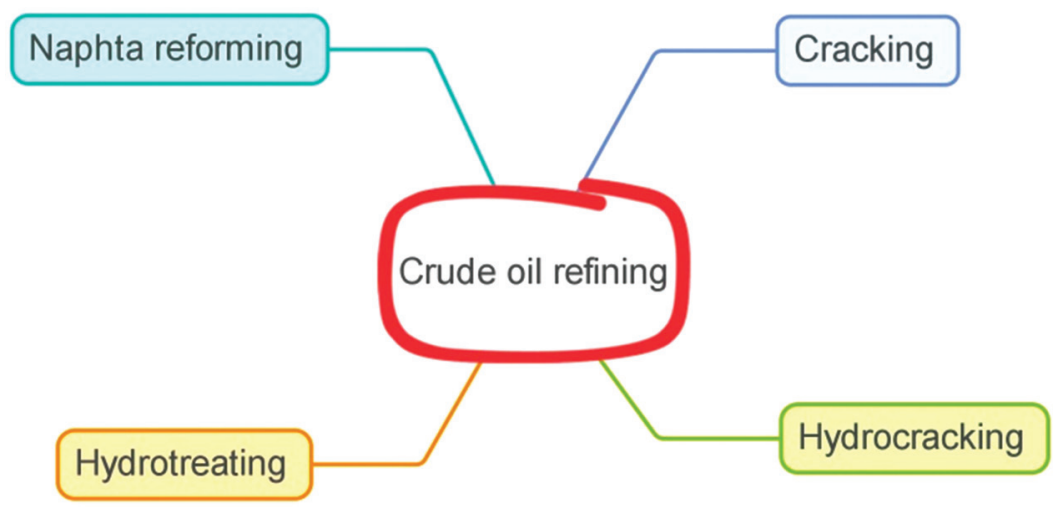

Figure 127. Nanocatalysts are used in four major processes in crude oil refining.

Cracking is the process in which the heavy molecules present in crude oil are broken down into smaller molecules. Hydrocracking is a cracking process in the presence of hydrogen. Hydrocracking is used when catalytic cracking is difficult. In the case of hydrotreating, extra hydrogen is used to remove contaminants. such as sulfur, nitrogen or metals. Naphta reforming is an important application of nanocatalysts because using nanosized platinum particles rather than larger particles greatly increases the efficiency of that process.

Nanocatalysis is also important in the fabrication of synthetic gas (syngas) which is a mixture of carbon monoxide and hydrogen. Syngas can be produced by several methods such as the gasification of coal, or steam reforming of natural gas. Transformations of the syngas are made using four main reactions: water-gas shift, methanation, methanol synthesis and Fischer-Tropsch synthesis.

Using nanocatalysts the water-shift reaction in which the carbon monoxide reacts with water vapor to produce carbon dioxide and hydrogen can be carried out at lower temperatures than with conventional catalysts. This is also true for methanol synthesis. Nanocatalysts are also employed to synthesize methane from the syngas (the methanation process). but the yield decreases as the nanoparticles of the catalysts grow and become larger.

Hydrocarbons can also be synthesized from syngas by means of the Fischer-Tropsch reaction. This process has been used at an industrial scale to synthesize fuel in situations where oil is not available (Germany during World War II, South Africa). Nanocatalysts can provide large increases in efficiency relative to conventional catalyst systems. 


\section{Renewable energies}

Renewable energies are strongly developing globally but their contribution to the global energy consumption remains small. The renewable energy consumption per inhabitant per year has remained almost constant for centuries. It is about 0.2 Gtoe. Biomass is the renewable energy source which is mostly used and hydro power to produce electricity is second. Wind and solar energies are currently swiftly developing because of incentives, but their intermittency is a serious issue. They need either large scale storage facilities or associated gas or coal fired plants to provide electricity when there is not enough wind or sun. Smart grids can help to some extent. So far there is no easy and inexpensive solution to wind or solar energy intermittency.

\section{Solar energy}

Solar energy can be transformed into electricity using photovoltaic cells. They are made with different semiconducting materials. Most of them are made out of silicon either in the crystalline or polycrystalline form. A second generation of cells has been developed based on thin films (cadmium telluride, copper indium selenium diselenide, amorphous silicon). Their efficiencies are smaller but their cost and the energy needed to manufacture them is also smaller. Nanotechnology is already involved in the development of photovoltaic cells and a research effort is underway to develop a third generation of cells with a high-efficiency conversion based on nanostructured materials. In particular, nanocrystal (quantum dots) technologies improve the efficiency of solar cells because they can be tailored to harvest a greater part of the solar energy spectrum. It has been found, in the USA, that PbSe nanoparticles with a diameter smaller than $10 \mathrm{~nm}$ have the ability to generate up to 3 electrons for each absorbed photon. In the solar cells used today, only one electron is produced by an absorbed photon. Multiple electron production can boost the efficiency of photovoltaic solar cells.

Organic photovoltaic cells are also an interesting way to harness solar energy. They are based on dyes. They can be either completely organic or contain mineral components like dye-sensitized photoelectrochemical cells (Grätzel cells). Molecular engineering is essential to find efficient dyes and self-assembly techniques are needed to build the device. Today their 
yields are small and the life times short. Component aging is a great issue in this field but progress is being made.

In all new developments of solar photovoltaic cells, nanotechnology is widely used, either in the form of nanosized objects (nanocrystals, nanoparticles, quantum dots, etc.), or in the nanostructuration of the material. It is possible to manufacture a photoactive nanoscale material which can be printed or painted on to various surfaces. If that is a flexible plastic foil solar cells can be manufactured in rolls. The goal is to provide flexible, low-cost photovoltaic cells that can be manufactured by such simple techniques.

Harvesting solar heat is also a way to harness the energy coming from the sun. Nanostructured mirrors and lenses allow a better solar thermal collection. Aerogels, with nanopores which are both transparent to solar light and good thermal insulators can be used to improve solar collectors. Nanoadditives, nanoparticles or nanopowders can enhance the heat transfer from the collectors to the heat reservoir tank

\section{Wind energy}

Wind energy is harnessed with large wind turbines. The power delivered by a wind turbine is proportional to the cube of the wind velocity. Therefore, the delivered power strongly increases with the wind velocity and the blades are subject to severe mechanical constraints. Wind turbines must even be stopped if the speed of the wind becomes too large (typically larger than $90 \mathrm{~km} / \mathrm{h}$ ). Carbon nanotubes bound in epoxy can be used to make stronger and lighter blades. They can be $50 \%$ lighter than glass fiber blades and the yield of the wind turbine can be increased by $30 \%$.

\section{Energy storage}

Energy storage, and, in particular electricity storage, is the weak point of the energy sector. It is necessary to store widely varying amounts of electrical energy ranging from milliwatt hours for small portable devices, to terawatt hours, for the grid. Smaller energy amounts are also needed for microdevices. The development of intermittent renewable energies requires the development of efficient storage capacities otherwise other sources are needed to produce electricity when there is not enough, wind or sun. 


\section{Batteries}

Among electrical storage devices, rechargeable batteries are particularly important. In a battery, electrical energy is stored in the form of chemical energy. When the battery runs, the chemical energy is transformed into electrical energy through a redox reaction taking place at the electrodes of the battery (except in the case of li-ion batteries where insertion processes take place). The positive electrode captures electrons and the negative electrode releases them. Positive and negative electrodes face each other and are separated by an ionic electrolyte allowing the motion of ions from one electrode to the other.

A battery is usually made of several individual cells associated in series or in parallel to provide the desired voltage and power. The electrodes are porous complex composite systems where nanomaterials and nanostructuration are essential. Carbon black or carbon nanotubes can be used. Nanotechnology is also applied to the development of the electrolyte separating the two electrodes.

Nanotechnology is used in batteries, especially in lithium-ion batteries, to provide increased energy densities with decent recharge times. In Li-ion batteries, the lithium ion $\mathrm{Li}^{+}$is used and at least one electrode is an open crystal structure with empty spaces to intercalate $\mathrm{Li}^{+}$ions. There are several Li-Ion technologies. For example, in $\mathrm{LiCoO}_{2}$ batteries, the oxidation of Co expels the $\mathrm{Li}^{+}$ion which is intercalated in the graphite electrode. During charging, the $\mathrm{Li}^{+}$ion moves from the positive electrode to the negative one in the electrolyte. During the discharge, the opposite mechanism takes place. The main issue is to have a good reversibility in the process taking place at the nanoscale.

Fast charging and discharging is an important quality of a battery which can be obtained if the active materials of the electrodes are thin films. However, this limits the energy capacity of the battery because of the limited amount of active material. Increasing the energy capacity necessitates thicker films and results in slower charging properties. The question of power of the battery is also an issue. Power is related to the ion removal capability which in turns depends on the electrochemical properties of the battery.

\section{Supercapacitors}

Supercapacitors provide another way to store electricity. Their energy densities are intermediate between those of rechargeable batteries and 
electrochemical capacitors. One of their advantages is their high power densities (about 10 times that of a battery) and the fact that they can make a very large number of charge-discharge cycles compared to batteries.

Nanotechnology can be applied to increase the performance of supercapacitors. The power density of a supercapacitor depends especially on the nature and structure of the electrodes. Carbon in different forms is the most frequently used electrode material. Single-walled and multi-walled nanotubes of carbon have the ability to increase the power density of supercapacitors. In the laboratory, an increase of a factor of seven has been measured compared to conventional electrodes.

Construction of supercapacitors with a $3 \mathrm{D}$ nano-architecture in which positive and negative electrodes interpenetrate while being electrically separated from each other is a feasible goal. Paper batteries, combining nanotubes and cellulose layers, have recently been developed in a US laboratory. This opens the way to manufacturing techniques based on rolling and folding.

\section{Electricity}

Electricity is an essential energy vector. The need for electricity is ever increasing. Electricity has to be transported from the producer to the consumer and there are losses. Due to the joule effect: part of the initial energy carried by the grid is lost as heat. The amount of electricity lost in the grid is about $7-10 \%$. The use of high critical temperature superconducting materials at is a way to reduce the losses in part of the grid. Nanotechnology can be used to tailor interfaces and multilayers of the superconducting materials. Presently superconducting cables maintained at the temperature of liquid nitrogen, $77 \mathrm{~K}$, exist and a few commercial installations have been completed worldwide.

Another challenge is to develop a light, low energy loss material to replace the copper cables used today. Carbon nanotubes can meet this demand because they have a better electrical conduction than copper and their weight is about one fifth that of this metal. Furthermore, their mechanical strength is much larger. Positive results for this material have been obtained in the laboratory.

\section{Hydrogen}

Hydrogen is also an energy vector which can play an important role in the future. It can provide an alternative way to store electricity from intermittent 
renewable energies sources such as wind or solar energies. In this case electricity produced by these sources is used to electrolyze water and produce hydrogen. Electrolysis of water is not economically competitive compared to hydrogen production from natural gas by vaporeforming. However, in the case of intermittent energy sources it is a possible solution.

Photoelectrochemical water splitting is another route to produce hydrogen from water. Nanosized semiconductor particles provide the best efficiency to perform this operation.

Hydrogen storage is an important issue. A characteristic of hydrogen is that its energy density per unit of mass is about 3 times that of gasoline. However, in the area of transportation, the key parameter is the energy density per unit of volume and the figure is not as good. Hydrogen stored at 800 bars ( 800 times the atmospheric pressure) has about 5 times less energy density per unit of volume than gasoline. Liquid hydrogen has a similar energy density per unit of volume to that of compressed hydrogen at 800 bars but requires energy to be liquefied from the gas (theoretically, about $4 \mathrm{kWh} / \mathrm{kg}$ are required).

Solid-state storage of hydrogen is an interesting possibility. This can be done using metal hydrides. For example, by forming metal hydrides certain alloys of magnesium have the ability to absorb large quantities of hydrogen. Hydrogen can also be stored by chemisorption or physisorption in carbon nanotubes. Theoretically, single-walled nanotubes can absorb $14 \%$ by weight of hydrogen and multi-walled nanotubes about $7.7 \%$ by weight.

The main issues for hydrogen stored in solid metallic supports are the material loading capacity and the speed at which it is possible to extract hydrogen from the containing matrix. Nanotechnology is of interest in this respect because of the high surface-to-volume ratios existing in nanomaterials. Nanostructured materials, such as activated carbon, can be made extremely porous with tiny cavities which are able to store hydrogen. In all solid-storage solutions, a cycle for charge and discharge of hydrogen is needed.

\section{Fuel cells}

Most fuel cell employ hydrogen as an energy vector. Such cells are used to convert the chemical energy of hydrogen into electricity. The chemical reaction in the fuel cell is basically the inverse of what happens in water electrolysis. Hydrogen reacts with oxygen contained in the air to form water. There are several fuel cell technologies as indicated in figure 128 . 


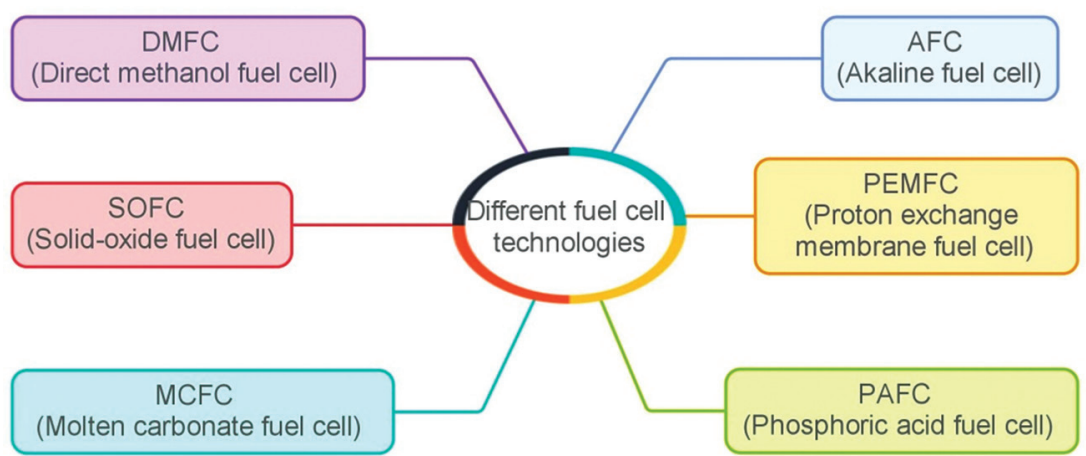

Figure 128. Different fuel cell technologies used to generate electricity.

Except for the DMFC technology using methanol as fuel, other technologies use hydrogen. The most promising ones for large scale applications are the PEMFC for vehicles, and the SOFC for heat and electricity production in buildings. The DMFC technology is interesting for portable devices such as laptop computers or mobile phones. Nanotechnology is essential to progress in the development of fuel cells. Nanostructured materials improve the characteristics of the membranes and electrodes which are crucial parts of fuel cells. Some fuel cell technologies need a catalyst to function. As already noted, nanoparticles provide very large areas in contact with the reactants, increasing catalytic activity. Platinum is widely used but scientists are also trying to find other catalysts that are not noble metals because a large scale deployment of fuel cells could be hampered by a shortage of noble metals. For example, suppose that all cars in world were to be equipped with a PEMFC. With the present quantity of platinum required for the catalyst, a quantity corresponding to almost 300 years of the yearly world production of platinum would be needed.

\section{Thermoelectricity}

Using thermoelectric techniques, temperature differences between two appropriate materials can be exploited to produce electricity and vice versa. There are three kinds of thermoelectric effects:

- The Seebeck effect, discovered in the early 180os, occurs when two different materials in contact are at a different temperature. A voltage difference proportional to the temperature difference is observed. The coefficient of proportionality is called the Seebeck coefficient. The thermocouple is a current application of the Seebeck effect. 
- The Peltier effect is just the contrary. An electrical current flowing between a junction of two appropriate conductors can generate heating or cooling. Cooling is usually the most desired effect for applications.

- The Thomson effect occurs in one material where a thermal gradient is present. If an electrical current is applied, heat can be produced. If a heat flux exists, an electrical current is produced.

Currently, part of sun's radiation can be converted to electricity using photovoltaic cells. This corresponds to about $58 \%$ of the radiated energy (the UV and visible part of the light spectrum). The remaining part, $42 \%$, is in the infrared region (heat). Harvesting this infrared part is also desirable. This is possible employing the Seebeck effect. The efficiency of conversion depends on the nature of the materials used to make the junction. Semiconducting materials are particularly useful because it is possible to tune their electrical properties. Developing hybrid systems containing photovoltaic and thermoelectric cells delivers the ability to harness more energy from the sun.

Apart from solar thermoelectric conversion, thermoelectric devices are used in several niche applications such as producing electricity in deep -space missions, in regions where they do not receive much energy from the sun. The radioactive isotope, ${ }^{238} \mathrm{Pu}$, is used as a thermal energy source and SiGe as the thermoelectric junction. There are also applications such as those used to warm or cool seats in luxury cars.

If small amounts of energy are needed (wrist watches, pacemakers...) the small differences of temperature within the body, or between the body and the ambient temperature, is enough to operate a thermoelectric device.

The efficiency of thermoelectric devices is around $7 \%-8 \%$. A much better efficiency, in the range of $15-20 \%$ would make them useful in large scale applications. Using nanomaterials and nanostructuration, new thermoelectric devices can be developed. For example, energy could be recovered from the exhaust pipe of a car. In research employing nanostructured materials an efficiency increase of the order of $50 \%$ has been obtained.

\section{Nuclear energy}

Both the fission of heavy elements and the fusion of light elements can release large amounts of energy. The technology of the former is well in 
hand and nuclear fission reactors provide about $5 \%$ of our global primary energy. Fusion power is still in the research stage.

In current nuclear plants a large variety of materials are involved. But it is in the reactor vessel that materials are submitted to the most extreme conditions of temperature, pressure, radiation, chemical and mechanical stresses. Outside the reactor vessel there is, at a much lower level, also a degrading influence of radiation on concrete, cables, the corrosion of heat exchangers, etc.

Consider the materials used inside the reactor vessel, for example the cladding of the nuclear fuel. That fuel is enclosed in a leak tight housing. Basically, as the nuclear fuel is burned, the nature of the chemical composition changes and a few gaseous elements are produced. All the products must remain confined inside the housing in order not to contaminate the coolant flowing around the housing. The housing is submitted to high temperatures, high neutron radiation, and the physical transformation in the burned fuel induces strong mechanical constraints on the housing materials. This is amplified when a high burn up (i.e. when a lot of energy is extracted from the nuclear fuel) is demanded.

Mechanical and plastic properties of materials, such as metals or alloys, depend on the mobility of the linear defects (dislocations) in those materials. These dislocations impact the elasticity limit of the material and its overall mechanical behavior. In nanostructured materials, the increased number of interfaces can anchors dislocation and increase the strength of the material. Furthermore, the interfaces facilitate recombination of point defects created by particle irradiation. There are several ways to amplify the number of interfaces: added or precipitated nanoparticles, nanostructuration, thin films, superlattices, etc.

Nanostructured materials can be used to push the usage limits of conventional materials. This is useful to prepare the next generation of nuclear reactors based on fast neutrons. Some of them use sodium as a coolant and operate at temperatures in the range of $400-650^{\circ} \mathrm{C}$ under a pressure of a few bars. In a fast sodium nuclear reactor, the fissile fuel is in the form of pellets of mixed uranium dioxide and plutonium dioxide piled up in leaktight pins placed in sub-assemblies of stainless steel housing which are cooled by a sodium flow. New steels reinforced with oxides have been developed to suit these extreme operating conditions. They are called ODS-steels (oxide dispersion strengthened). ODS are metallic materials reinforced by nanosized oxide nanoparticles manufactured by powder metallurgy. This nanostructuration substantially improves the mechanical properties (breaking strength under tension, creeping, thermal stability). However, 
the anisotropy of the mechanical properties of ODS might prevent their industrial development. On the other hand, interesting results have been obtained with nanolayer systems possessing selfhealing properties. This technology is useful for the manufacture of "accident tolerant nuclear fuels" because they exhibit better radiation resistance during normal operation.

The development of high temperature gas reactors is another line of research. These are fast neutron reactors cooled by a gas (helium). They are operated at temperatures in the range of $500-85^{\circ} \mathrm{C}$, providing a good yield to produce electricity because of the Carnot principle. The fuel is the form of carbide and the cladding of nuclear fuel requires materials resistant at these very high temperatures. It is necessary to develop new ceramic materials to fulfill this requirement but this is still at an early research stage. At any rate, nanostructuration is essential. Silicon carbide is a possibility and, in particular, long fibers of $\mathrm{SiC}$ can be used to give good ductile properties.

Nanomaterials and nanostructured materials can also be expected to make important contributions in future nuclear fusion devices.

\section{Summary}

Nanomaterials, nanoparticles and nanostructuration play a role in many areas of energy production. They can substantially improve technologies based on conventional materials and processes. Nanotechnology will be essential in the development of future of solar cells. Nanocatalysts will continue to play a key role in the transformation processes converting primary energy sources to final energy sources available to the consumer. Energy storage capabilities, the weak link of the energy domain, will be improved using nanotechnology in the area of batteries, supercapacitors, etc. Fuel cells that use hydrogen to produce electricity will use nanotechnology to develop better membranes and catalysts. Finally, harnessing additional energy production techniques such as thermoelectricity will rely on nanotechnology to reach an industrial stage. Nanotechnology is expected to transform the energy domain over the next few decades. 